\title{
On the Sizes of Maximal Independent Sets of Cylindrical Grid Graphs
}

\author{
R.M. BARBOSA and M.R. CAPPELLE*
}

Received on September 11, 2014 / Accepted on November 28, 2016

\begin{abstract}
If a graph $G$ has exactly $t$ different sizes of maximal independent sets, $G$ belongs to a collection called $\mathcal{M}_{t}$. For the Cartesian product of the graph $P_{n}$, the path of length $n$, and $C_{m}$, the cycle of length $m$, called cylindrical grid, we present a method to find maximal independent sets having different sizes and a lower bound on $t$, such that these graphs belong to $\mathcal{M}_{t}$.
\end{abstract}

Keywords: Well-covered graph, maximal independent set, Cartesian product.

\section{INTRODUCTION}

In [9] Plummer defines a graph to be well-covered if all its maximal independent sets have the same size. Generalizing this concept, Finbow, Hartnell, and Whitehead [4] define, for every $t \in \mathbb{N}$, the set $\mathcal{M}_{t}$ as the set of graphs that have maximal independent sets of exactly $t$ different sizes. With this notation, $\mathcal{M}_{1}$ is precisely the set of well-covered graphs.

Well-covered graphs have been investigated from several different parameters. See the survey [10], for more details. Topp and Volkmann [11] proposed the following question about Cartesian products and well-covered graphs: "Do there exist non well-covered graphs whose Cartesian product is well-covered?". This question was partially answered by Fradkin [5] for some classes of triangle-free graphs and recently a negative answer was given by Hartnell and Rall [7], for arbitrary graph. For $G \in \mathcal{M}_{t}$ and $v \in V(G)$, Barbosa and Hartnell [2] determine the extreme values that $r$ can assume where $G \backslash v$ belongs to $\mathcal{M}_{r}$. Additional properties of graphs in this class are given in [1]. Results on $\mathcal{M}_{t}$, for $t \geq 2$, related to graphs without small cycles are also given in $[3,4,6]$.

The Cartesian product $P_{n} \square C_{m}$ is a cylindrical grid graph. Every maximal independent set in a graph is a dominating set, although the converse is not always true. In [8], methods to find the domination number of cylindrical grid graphs $P_{n} \square C_{m}$ with $m \geq 3$ and $n=2,3$, and 4 were proposed. Moreover, bounds on the domination numbers were found when $n=5$ and $m \geq 3$. We present a method to find maximal independent sets having different sizes and a lower bound for $t$, such that $P_{n} \square C_{m}$ belongs to $\mathcal{M}_{t}$.

*Corresponding author: Márcia Rodrigues Cappelle.

Instituto de Informática, Universidade Federal de Goiás, 74690-900 Goiânia, Brasil.

E-mails: rommel@inf.ufg.br; marcia@inf.ufg.br 
Before we present our results and proofs, we summarize our notation.

We consider finite, simple, and undirected graphs. For a graph $G$, the vertex set and the edge set are denoted $V(G)$ and $E(G)$, respectively. For a vertex $u$ of $G$, its neighbourhood is denoted $N_{G}(u)$ and its closed neighbourhood denoted $N_{G}[u]$ is the set $N_{G}(u) \cup\{u\}$. For a set $U$ of vertices of $G$, let

$$
N_{G}(U)=\bigcup_{u \in U} N_{G}(u) \backslash U \text { and } N_{G}[U]=N_{G}(U) \cup U .
$$

A set $D \subseteq V(G)$ of a graph $G$ is a dominating set if every vertex $v \in V(G) \backslash D$ is adjacent to some vertex $u \in D$. The domination number of $G$ is the cardinality of a smallest dominating set of the graph $G$ and is usually denoted by $\gamma(G)$.

A set $I$ of vertices of a graph $G$ is independent if no two vertices in $I$ are adjacent. An independent set $I$ of $G$ is maximal if every vertex $u$ in $V(G) \backslash I$ has a neighbour in $I$. An independent set $I$ of $G$ is maximum if $G$ has no independent set $J$ with $|J|>|I|$. We denote $i(G)$ the cardinality of a smallest maximal independent set in $G$. We denote $\alpha(G)$ the cardinality of a maximum independent set in $G$ and $m s(G)$ the set of all sizes of maximal independent sets in $G$. Hence, if $G$ is well-covered, then $i(G)=\alpha(G)$ and $|m s(G)|=1$.

For any two graphs $G$ and $H$, the Cartesian product $G \square H$ is the graph with vertex set $V(G) \times$ $V(H)$, such that two vertices $\left(u_{1}, v_{1}\right)$ and $\left(u_{2}, v_{2}\right)$ of $G \square H$ are adjacent whenever $v_{1}=v_{2}$ and $u_{1} u_{2} \in E(G)$ or $u_{1}=u_{2}$ and $v_{1} v_{2} \in E(H)$. Here, the vertices of the path $P_{n}$ or the cycle $C_{n}$ are always denoted $0,1, \ldots, n-1$. For the graph $P_{n} \square C_{m}$, we denote $\left(C_{m}\right)_{i}$ the graph $C_{m} \square\{i\}$, with $i \in V\left(P_{n}\right)$.

For naturals $a, b$, and $c$, with $c>b+1$, we shall denote the set $\{i: a \leq i \leq b\} \cup\{c\}$ by $\{a, \ldots, b, c\}$.

\section{RESULTS}

In [8] Nandi, Parui, and Adhikari establish the following result regarding the domination number of Cartesian product of paths and cycles.

Theorem 1. [8] For all $m \geq 3$,

- $\gamma\left(P_{2} \square C_{m}\right)= \begin{cases}\frac{m}{2}, & \text { if } m \equiv 0(\bmod 4), \\ \left\lceil\frac{m+1}{2}\right\rceil, & \text { otherwise; }\end{cases}$

- $\gamma\left(P_{3} \square C_{m}\right)=\left\lceil\frac{3 m}{4}\right\rceil$;

- $\gamma\left(P_{4} \square C_{m}\right)= \begin{cases}m+1, & \text { for } m=3,5,9, \\ m, & \text { otherwise; }\end{cases}$

- for $m \geq 6, m+\left\lceil\frac{m}{5}\right\rceil \leq \gamma\left(P_{5} \square C_{m}\right) \leq m+\left\lceil\frac{m}{4}\right\rceil$. 
One of our main results is the following. It will be proved in Section 2.2.

Theorem 2. Let $n \geq 3$ and $m \geq 4$. If $G=P_{n} \square C_{m}$, then $G \in \mathcal{M}_{t}$ for some

$$
t \geq \begin{cases}n\left\lfloor\frac{m}{4}\right\rfloor, & \text { if } m \equiv 0,2,3(\bmod 4), \\ n\left\lfloor\frac{m}{4}\right\rfloor-\left\lfloor\frac{n}{2}\right\rfloor, & \text { if } m \equiv 1(\bmod 4) .\end{cases}
$$

We begin showing the size of a maximum independent set in a graph $P_{n} \square C_{m}$.

Proposition 3. For $n \geq 2$ and $m \geq 3, \alpha\left(P_{n} \square C_{m}\right)=n\left\lfloor\frac{m}{2}\right\rfloor$.

Proof. Since $\alpha\left(C_{m}\right)=\left\lfloor\frac{m}{2}\right\rfloor$ and we have $n$ cycles $C_{m}$, we conclude that $\alpha\left(P_{n} \square C_{m}\right) \leq$ $n\left\lfloor\frac{m}{2}\right\rfloor$. Now, we show that $\alpha\left(P_{n} \square C_{m}\right) \geq n\left\lfloor\frac{m}{2}\right\rfloor$ presenting a maximal independent set with this cardinality. Let $I$ be the set of vertices $(i, j)$ of $P_{n} \square C_{m}$ such that $i=0, \ldots, n-1$, $j=0, \ldots, 2\left\lfloor\frac{m}{2}\right\rfloor-1$, and $i+j$ is an odd integer. The set $I$ has cardinality $n\left\lfloor\frac{m}{2}\right\rfloor$ and it is a maximal independent set in $P_{n} \square C_{m}$.

In Theorem 7, we determine $t$ such that $P_{2} \square C_{m}$ belongs to $\mathcal{M}_{t}$. Before, we prove some preliminary results.

Lemma 4. Let $m \geq 3$ and $G=P_{2} \square C_{m}$. If $I$ is a maximal independent set of $G$, then $I$ has even cardinality.

Proof. Let $I$ be a maximal independent set in $G, X_{i}=I \cap\left(C_{m}\right)_{i}$ and $x_{i}=\left|X_{i}\right|$, for $i=0,1$. We show that $x_{0}=x_{1}$. By symmetry, consider the subgraph induced by $\left(C_{m}\right)_{0} \backslash X_{0}$, denoted by $H$. The graph $H$ has exactly $x_{0}$ disjoint connected paths. We denote them by $P(k), k=1, \ldots, x_{0}$. If $|V(P(k))|>3$, for some $k \in\left\{1, \ldots, x_{0}\right\},\left(C_{m}\right)_{0} \backslash N_{G}\left[X_{0}\right]$ has some path with at least two vertices, which implies at least two consecutive vertices in $I \cap\left(C_{m}\right)_{1}$. Thus we may assume $|V(P(k))| \leq 3, k=1, \ldots, x_{0}$.

Fix some $k \in\left\{1, \ldots, x_{0}\right\}$. Let $\left(v_{1}, v_{2}, \ldots, v_{q}\right)$, with $q=|V(P(k))|$, be one of the two orderings of the vertices of $P(k)$ such that adjacent vertices are consecutive. For each $j \in$ $\{1,2, \ldots,|V(P(k))|\}$, let $v_{j}^{\prime}$ be the neighbour of $v_{j}$ in $\left(C_{m}\right)_{1}$. Note that exactly $x_{0}$ vertices in $\left(C_{m}\right)_{1}$ are in $N_{G}\left(X_{0}\right)$ and they are separated by at most three vertices. We have three cases for each $P(k)$. If $|V(P(k))|=1, v_{1}^{\prime}$ must belong to $I$ since its neighbours in the same cycle are dominated by $I$. If $|V(P(k))|=2, v_{1}$ and $v_{2}$ belong to $N_{G}(I)$ and exactly one of $v_{1}^{\prime}$ and $v_{2}^{\prime}$ must belong to $I$. If $|V(P(k))|=3$, the vertices $v_{2}, v_{1}^{\prime}$, $v_{2}^{\prime}$, and $v_{3}^{\prime}$ are not in $N_{G}\left(X_{0}\right)$. Hence $v_{2}^{\prime}$ must be in $I$. Therefore, in all cases, for $k=1, \ldots, x_{0}$, for every path $P(k)$, there is exactly one vertex in $X_{1}$, resulting in $\left|I \cap\left(C_{m}\right)_{0}\right|=\left|I \cap\left(C_{m}\right)_{1}\right|$.

Lemma 5. Let $n \geq 2, m \geq 3$ and $G=P_{n} \square C_{m}$. If $I$ is a maximal independent set in $G$, then

$$
\left|I \cap\left(C_{m}\right)_{i}\right| \geq\left\lceil\frac{m}{4}\right\rceil
$$

for $i \in\{0, n-1\}$. 
Proof. Let $I$ be a maximal independent set in $G, X_{i}=I \cap\left(C_{m}\right)_{i}$ and $x_{i}=\left|X_{i}\right|$, for $i \in\{0, n-1\}$. Suppose $x_{i}<\left\lceil\frac{m}{4}\right\rceil$, for some $i \in\{0, n-1\}$. By symmetry, we may assume $i=0$. Consider the graph $\left(C_{m}\right)_{0}$ and the subgraph $H$ induced by $\left(C_{m}\right)_{0} \backslash N_{G}\left[X_{0}\right]$. Since at most $3\left\lceil\frac{m}{4}\right\rceil-3$ vertices of $\left(C_{m}\right)_{0}$ are in $N_{G}\left[X_{0}\right]$, at least $\left\lceil\frac{m}{4}\right\rceil$ of those vertices are not in $N_{G}\left[X_{0}\right]$. Moreover, $H$ has at most $x_{0}$ disjoint connected paths. Therefore, at least one of these paths has at least two vertices. Since $I$ is maximal, two adjacent vertices in $\left(C_{m}\right)_{1}$ are in $I$. This contradicts the independence of $I$ and completes the proof.

Proposition 6. For $m \geq 3, i\left(P_{2} \square C_{m}\right)=2\left\lceil\frac{m}{4}\right\rceil$.

Proof. Let $J=\{(0, i): i \equiv 1(\bmod 4)\} \cup\{(1, i): i \equiv 3(\bmod 4)\}$. We consider a set $I$ with $I=J$, if $m \equiv 0(\bmod 4), I=J \cup\{(1,0),(0, m-1)\}$, if $m \equiv 1(\bmod 4)$, and $I=J \cup\{(1,0)\}$, otherwise.

Note that $I$ is a maximal independent set of $P_{2} \square C_{m}$, and $|I|=2\left\lceil\frac{m}{4}\right\rceil$. Hence, $i\left(P_{2} \square C_{m}\right) \leq$ $2\left\lceil\frac{m}{4}\right\rceil$. By Lemma 5, $\left|I \cap\left(C_{m}\right)_{0}\right|+\left|I \cap\left(C_{m}\right)_{1}\right| \geq 2\left\lceil\frac{m}{4}\right\rceil$ and the desired statement follows.

Now, we can show the quantity of different sizes of maximal independent sets in $P_{2} \square C_{m}$.

Theorem 7. For $m \geq 3$ and $G=P_{2} \square C_{m}, G \in \begin{cases}\mathcal{M}_{\left\lfloor\frac{m}{4}\right\rfloor}, & \text { if } m \equiv 1(\bmod 4), \\ \mathcal{M}_{\left\lfloor\frac{m}{4}\right\rfloor+1}, & \text { otherwise. }\end{cases}$

Proof. By Proposition 3, $\alpha(G)=2\left\lfloor\frac{m}{2}\right\rfloor$, and by Proposition 6, $i(G)=2\left\lceil\frac{m}{4}\right\rceil$. We present maximal independent sets of $G$ with every even cardinality between $2\left\lceil\frac{m}{4}\right\rceil$ and $2\left\lfloor\frac{m}{2}\right\rfloor$, in view of Lemma 4.

First, let $\quad l= \begin{cases}m, & \text { if } m \equiv 0,3(\bmod 4), \\ m-3, & \text { if } m \equiv 1(\bmod 4), \\ m-2, & \text { if } m \equiv 2(\bmod 4),\end{cases}$

and

$$
k= \begin{cases}\left\lfloor\frac{m}{4}\right\rfloor-1, & \text { if } m \equiv 1(\bmod 4) \\ \left\lfloor\frac{m}{4}\right\rfloor, & \text { otherwise }\end{cases}
$$

Let $\left.I(0)^{\prime}=\{(1, i), i \equiv 0(\bmod 4)\}, i \leq l\right\} \cup\{(0, i), i \equiv 2(\bmod 4), i \leq l\}$ and

$$
I(0)= \begin{cases}I(0)^{\prime}, & \text { if } m \equiv 0,3(\bmod 4), \\ I(0)^{\prime} \cup\{(0, m-1),(1, m-2)\}, & \text { if } m \equiv 1(\bmod 4), \\ I(0)^{\prime} \cup\{(0, m-1)\}, & \text { if } m \equiv 2(\bmod 4)\end{cases}
$$

Note that the set $I(0)$ is a maximal independent set in $G$ and $|I(0)|=2\left\lceil\frac{m}{4}\right\rceil=i(G)$. For every $j \in\{1, \ldots, k\}$, let

$$
I(j)=I(j-1) \backslash\{(0,4 j-2)\} \cup\{(0,4 j-3),(0,4 j-1),(1,4 j-2)\} .
$$


For every $j \in\{0, \ldots, k\}, I(j)$ is a maximal independent set in $G$ and $|I(j)|=2\left\lceil\frac{m}{4}\right\rceil+2 j$.

Hence $G$ has maximal independent sets of $k+1$ different sizes which results in $\left\lfloor\frac{m}{4}\right\rfloor$ if $1 \equiv$ $m(\bmod 4)$, or $\left\lfloor\frac{m}{4}\right\rfloor+1$, otherwise. This completes the proof.

By Theorem 2, when $n \geq 3$ and $m \geq 4, P_{n} \square C_{m}$ is not well-covered. The graph $P_{2} \square C_{5}$ is well-covered and also $P_{n} \square C_{3}$ for $n \geq 2$, as we show in next proposition.

Proposition 8. For $n \geq 2$, if $G=P_{n} \square C_{3}$, then $G$ is well-covered.

Proof. By Proposition 3, $\alpha(G)=n$. Let $I$ be a maximal independent set in $G$. We show that $|I|=n$. For a contradiction, suppose $|I|<n$. Hence there is some $j \in\{0, \ldots, n-1\}$, with $I \cap\left(C_{3}\right)_{j}=\emptyset$. Let $S$ denote the set $N_{G}\left(V\left(\left(C_{3}\right)_{j}\right)\right)$. Since $I$ is maximal and from the structure of $G,|S \cap I| \geq 3$. But $S$ induces one or two cycles $C_{3}$, which contradicts the independence of $I$.

Proposition 9. Let $n \geq 3, m \geq 4$ and $G=P_{n} \square C_{m}$. If $m$ is even, then there is no maximal independent set $I$ in $G$ such that $|I|=\frac{n m}{2}-1$;

Proof. By Proposition 3, $\alpha(G)=\frac{n m}{2}$. A maximum independent set of $G$ has $\frac{m}{2}$ vertices of every cycle $\left(C_{m}\right)_{i}$, for $i=0, \ldots, n-1$. Suppose that there exists a maximal independent set $I$ of $G$ of size $\frac{n m}{2}-1$. Any independent set of $G$ has at most $\frac{m}{2}$ vertices on any cycle $\left(C_{m}\right)_{i}$, for $i=0, \ldots, n-1$. Hence there exists some $i \in\{0, \ldots, n-1\}$ such that $\left|\left(C_{m}\right)_{i} \cap I\right|=\frac{m}{2}-1$. At least one between $\left(C_{m}\right)_{i-1}$ and $\left(C_{m}\right)_{i+1}$ exists. Let $j \in\{i-1, i+1\}$ such that $\left(C_{m}\right)_{j}$ exists. Set $\left(C_{m}\right)_{j} \cap I$ has size $\frac{m}{2}$. Therefore, either all vertices $(j, k)$ with $k$ even or all vertices $(j, k)$ with $k$ odd are in $\left(C_{m}\right)_{j} \cap I$. Adjust notation of the vertex labeling of $C_{m}$ such that all vertices $(j, k)$ with $k$ even are in $I$. Therefore, all vertices $(i, f)$ of $\left(C_{m}\right)_{i} \cap I$ will be such that $f$ is odd. Considering $m \geq 4, \frac{m}{2}-1$ is at least one, thus $\left(C_{m}\right)_{i} \cap I$ has at least one vertex $(i, f)$ with $f$ odd. Therefore, if both $\left(C_{m}\right)_{i-1}$ and $\left(C_{m}\right)_{i+1}$ exist, then on both of them all vertices $(i-1, k)$ and $(i+1, k)$ for $k$ even are in $I$. Considering there is exactly $\frac{m}{2}-1$ vertices in $\left(C_{m}\right)_{i} \cap I$, there is a vertex $(i, f)$ with $f$ odd without a neighbour in $\left(C_{m}\right)_{i} \cap I$. However, $(i, f)$ is not a neighbour of any vertex in $I$. Thus, $I$ is not maximal.

\subsection{Two useful classes of graphs}

Before we prove Theorem 2, we show how to construct recursively two useful classes of graphs, denoted $H_{r}$ and $F_{s}$. Furthermore, we show the different sizes of maximal independent sets in these graphs. 
Let the graph $H_{1}$ have the vertex set $V^{(1)}=\left\{v_{1}^{(1)}, v_{2}^{(1)}, v_{3}^{(1)}\right\}$ and the edge set $E^{(1)}=\left\{v_{1}^{(1)} v_{2}^{(1)}\right.$, $\left.v_{2}^{(1)} v_{3}^{(1)}\right\}$. For $r \geq 2$, let the graph $H_{r}$ have the vertex set

$$
\left\{\begin{array}{lll}
V^{(r)}=V^{(r-1)} \cup\left\{v_{1}^{(r)}\right\}, & & \text { for } r \text { even } \\
V^{(r)}=V^{(r-1)} \cup\left\{v_{1}^{(r)}, v_{2}^{(r)}, v_{3}^{(r)}\right\}, & & \text { for } r \text { odd }
\end{array}\right.
$$

and the edge set

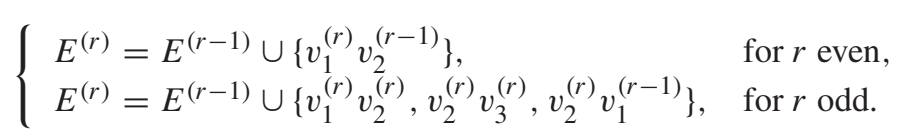

See Figure 1 for an example.



Figure 1: The graph $\mathrm{H}_{5}$.

From the recursive construction, we can determine the sizes of maximal independent sets in $H_{r}$, as we prove in Proposition 10.

Proposition 10. For $r \geq 2, H_{r} \in \mathcal{M}_{r}$. Furthermore, $\operatorname{ms}\left(H_{r}\right)=\left\{\left\lceil\frac{r}{2}\right\rceil, \ldots,\left\lceil\frac{3 r}{2}\right\rceil-2,\left\lceil\frac{3 r}{2}\right\rceil\right\}$.

Proof. We prove the statement by induction on $r$. For $r=2,3,4$, the result is trivial. Now let $r \geq 5$. We have to consider two cases. If $r$ is even, by construction of $H_{r}$, it was obtained from $H_{r-1}$ by the addition of the vertex $v_{1}^{(r)}$ and the edge $v_{1}^{(r)} v_{2}^{(r-1)}$. Since $v_{1}^{(r)}$ is a leaf, every maximal independent set in $H_{r}$ contains either $v_{1}^{(r)}$ or $v_{2}^{(r-1)}$. Considering the first case, $H_{r} \backslash N_{G}\left[v_{1}^{(r)}\right]$ has a component isomorphic to $H_{r-2}$ and two isolated vertices. By induction hypothesis, $H_{r-2} \in$ $\mathcal{M}_{r-2}$ and $\operatorname{ms}\left(H_{r-2}\right)=\left\{\frac{r}{2}-1, \ldots, \frac{3 r}{2}-5, \frac{3 r}{2}-3\right\}$. By adding the isolated vertices and $v_{1}^{(r)}$ we obtain maximal independent sets in $H_{r}$ with sizes in $A=\left\{\frac{r}{2}+2, \ldots, \frac{3 r}{2}-2, \frac{3 r}{2}\right\}$. The graph $H_{r} \backslash N_{G}\left[v_{2}^{(r-1)}\right]$ is isomorphic to $H_{r-3}$. By induction hypothesis, $H_{r-3} \in \mathcal{M}_{r-3}$ and $m s\left(H_{r-3}\right)=\left\{\frac{r}{2}-1, \ldots, \frac{3 r}{2}-6, \frac{3 r}{2}-4\right\}$. By adding $v_{2}^{(r-1)}$ we obtain maximal independent sets in $H_{r}$ with orders in $B=\left\{\frac{r}{2}, \ldots, \frac{3 r}{2}-5, \frac{3 r}{2}-3\right\}$. Since $A \cup B=\operatorname{ms}\left(H_{r}\right)=\left\{\frac{r}{2}, \ldots, \frac{3 r}{2}-2, \frac{3 r}{2}\right\}$, the desired statement follows for $r$ even, with $r \geq 5$.

If $r$ is odd, by construction of $H_{r}$, it was obtained from $H_{r-1}$ by the addition of the vertices $v_{1}^{(r)}, v_{2}^{(r)}$ and $v_{3}^{(r)}$, and the edges $v_{1}^{(r)} v_{2}^{(r)}, v_{2}^{(r)} v_{3}^{(r)}$ and $v_{2}^{(r)} v_{1}^{(r-1)}$. Since $v_{1}^{(r)}$ and $v_{3}^{(r)}$ have degree one, every maximal independent set in $H_{n}$ contains either $v_{1}^{(r)}$ and $v_{3}^{(r)}$, or $v_{2}^{(r)}$. Considering the first case, $H_{r} \backslash N_{G}\left[\left\{v_{1}^{(r)}, v_{3}^{(r)}\right\}\right]$ is isomorphic to $H_{r-1}$. By induction hypothesis, $H_{r-1} \in \mathcal{M}_{r-1}$ 
and $\operatorname{ms}\left(H_{r-1}\right)=\left\{\frac{r-1}{2}, \ldots, \frac{3 r-1}{2}-3, \frac{3 r-1}{2}-1\right\}$. By adding $v_{1}^{(r)}$ and $v_{3}^{(r)}$ we have maximal independent sets in $H_{n}$ with sizes in $C=\left\{\frac{r+1}{2}+1, \ldots, \frac{3 r+1}{2}-2, \frac{3 r+1}{2}\right\}$. The graph $H_{r} \backslash$ $N_{G}\left[v_{2}^{(r)}\right]$ is isomorphic to $H_{r-2}$. By induction hypothesis, $H_{r-2} \in \mathcal{M}_{r-2}$ and $\operatorname{ms}\left(H_{r-2}\right)=$ $\left\{\frac{r-1}{2}, \ldots, \frac{3 r+1}{2}-5, \frac{3 r+1}{2}-3\right\}$. By adding the vertex $v_{2}^{(r)}$ we obtain maximal independent sets in $H_{r}$ with sizes in $D=\left\{\frac{r+1}{2}, \ldots, \frac{3 r+1}{2}-4, \frac{3 r+1}{2}-2\right\}$. Since $C \cup D=\operatorname{ms}\left(H_{r}\right)=\left\{\frac{r+1}{2}, \ldots, \frac{3 r+1}{2}-\right.$ $\left.2, \frac{3 r+1}{2}\right\}$, the desired statement follows for $r$ odd, with $r \geq 5$. This completes the proof.

Proposition 11. Let $r$ be a fixed positive integer with $r \geq 3$ and $t \geq 1$. If $G$ is a graph with $t$ components, each one isomorphic to $H_{r}$, then $G \in \mathcal{M}_{r t}$ and,

$$
\operatorname{ms}(G)=\left\{t\left\lceil\frac{r}{2}\right\rceil, \ldots, t\left(\left\lceil\frac{r}{2}\right\rceil+r\right)-2, t\left(\left\lceil\frac{r}{2}\right\rceil+r\right)\right\} .
$$

Proof. For $t=1$ the result is trivial in view of Proposition 10. We consider $t \geq 2$. Let $L_{j}$, for $j \in\{1, \ldots, t\}$, be the components of $G$. Let $I(j)$ be a maximal independent set in the subgraph $L_{j}$. For every $j \in\{1, \ldots, t\}, \bigcup_{j=1}^{t} I(j)$ is a maximal independent set of $G$. By Proposition 10, $|I(j)| \in\left\{\left\lceil\frac{r}{2}\right\rceil, \ldots,\left\lceil\frac{r}{2}\right\rceil+r-2,\left\lceil\frac{r}{2}\right\rceil+r\right\}$. Thus, all values on the interval $\left[\left\lceil\frac{r}{2}\right\rceil,\left\lceil\frac{r}{2}\right\rceil+r-2\right]$ are present in $\mathrm{ms}\left(L_{j}\right)$. Therefore, it is possible to obtain a maximal independent set $I$ of $G$ with $|I|=x$ for all $x$ in the interval $\left[t\left\lceil\frac{r}{2}\right\rceil, t\left(\left\lceil\frac{r}{2}\right\rceil+r-2\right)\right]$, through the combination of maximal independent sets of graphs $L_{j}$. We are still missing all integers on the interval $\left[t\left(\left\lceil\frac{r}{2}\right\rceil+r-2\right)+1, t\left(\left\lceil\frac{r}{2}\right\rceil+r\right)-2\right]$ and $t\left(\left\lceil\frac{r}{2}\right\rceil+r\right)$ for $\operatorname{ms}(G)$. For any integer $k \in\{0, \ldots, t\}$, it is possible to combine $k$ maximal independent sets of $L_{j^{\prime}}, j^{\prime}=0, \ldots, k$, of size $\left\lceil\frac{r}{2}\right\rceil+r-2$ with $t-k$ maximal independent sets of the remaining $L_{j^{\prime \prime}}, j^{\prime \prime}=k+1, \ldots, t$, with size $\left\lceil\frac{r}{2}\right\rceil+r$, resulting in a maximal independent set of $G$ with size $t\left(\left\lceil\frac{r}{2}\right\rceil+r\right)-2 k$. Thus, all integers on the set $\left\{t\left(\left\lceil\frac{r}{2}\right\rceil+r\right)-2 k: 0 \leq k \leq t\right\}$ are also on the set $\operatorname{ms}(G)$. For $r \geq 3$, both $\left(\left\lceil\frac{r}{2}\right\rceil+r-2\right)$ and $\left(\left\lceil\frac{r}{2}\right\rceil+r-3\right)$ belong to $\mathrm{ms}\left(L_{j}\right)$. So, by taking one maximal independent set of $L_{1}$ with size $\left\lceil\frac{r}{2}\right\rceil+r-3, k-1$ maximal independent sets of $L_{j^{\prime}}, j^{\prime}=2, \ldots, k$, of size $\left\lceil\frac{r}{2}\right\rceil+r-2$, and $t-k$ maximal independent sets of $L_{j^{\prime \prime}}, j^{\prime \prime}=k+1, \ldots, t$, with size $\left\lceil\frac{r}{2}\right\rceil+r$, we obtain a maximal independent set of $G$ with size $t\left(\left\lceil\frac{r}{2}\right\rceil+r\right)-2 k-1$, for $k=1, \ldots, t$. Therefore, $\operatorname{ms}(G)=\left\{t\left\lceil\frac{r}{2}\right\rceil, \ldots, t\left(\left\lceil\frac{r}{2}\right\rceil+r\right)-2, t\left(\left\lceil\frac{r}{2}\right\rceil+r\right)\right\}$.

Now we show how to construct a graph $F_{s}$, recursively.

Let the graph $F_{1}$ have the vertex set $V^{(1)}=\left\{u_{1}^{(1)}, u_{2}^{(1)}\right\}$ and the edge set $E^{(1)}=\left\{u_{1}^{(1)} u_{2}^{(1)}\right\}$. For $s \geq 2$, let the graph $F_{s}$ have the vertex set $V^{(s)}=V^{(s-1)} \cup\left\{u_{1}^{(s)}, u_{2}^{(s)}\right\}$ and the edge set

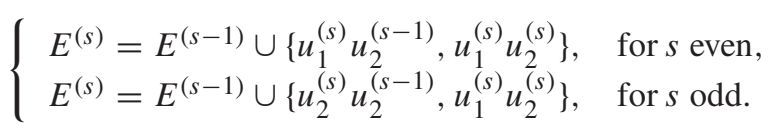

Proposition 12. For $s \geq 1$, the graph $F_{s} \in \mathcal{M}_{\left\lceil\frac{s}{2}\right\rceil}$ and $\operatorname{ms}\left(F_{s}\right)=\left\{\left\lfloor\frac{s}{2}\right\rfloor+1, \ldots, s\right\}$. 
Proof. By construction, $F_{s}$ has $\left\lfloor\frac{s}{2}\right\rfloor+1$ vertices of degree one. Moreover, no pair of such vertices share a neighbour. Therefore, $i\left(F_{s}\right) \geq\left\lfloor\frac{s}{2}\right\rfloor+1$. For every $i=1, \ldots, s$, at most one vertex of $\left\{u_{1}^{(i)}, u_{2}^{(i)}\right\}$ can be in a maximal independent set of $F_{s}$, thus $\alpha\left(F_{s}\right) \leq s$. We conclude the proof showing how to find in $F_{s}$ maximal independent sets with sizes in the set $\left\{\left\lfloor\frac{s}{2}\right\rfloor+1, \ldots, s\right\}$. Let $I(0)$ be the set containing, for $i=0, \ldots,\left\lceil\frac{s}{2}\right\rceil-1$, the vertices $u_{2}^{(2 i+1)}$. If $s$ is even, the vertex $u_{2}^{(s)}$ must be added to $I(0)$. Note that $I(0)$ is a maximal independent set in $F_{s}$. For every $j \in\left\{1, \ldots,\left\lceil\frac{s}{2}\right\rceil-1\right\}$, let $I(j)=\left(I(j-1) \backslash u_{2}^{(2 j-1)}\right) \cup\left\{u_{1}^{(2 j-1)}, u_{1}^{(2 j)}\right\}$.

For $j=0, \ldots,\left\lceil\frac{s}{2}\right\rceil-1, I(j)$ is a maximal independent set in $F_{s}$ and $|I(j)|=\left\lfloor\frac{s}{2}\right\rfloor+j+1$, which implies $m s\left(F_{S}\right)=\left\{\left\lfloor\frac{s}{2}\right\rfloor+1, \ldots, s\right\}$.

\subsection{Proof of Theorem 2}

Now we use the graphs $H_{n}$ and $F_{n}$ and their different sizes of maximal independent sets to show a lower bound on the number of possible sizes of maximal independent sets in $P_{n} \square C_{m}$, for $n \geq 3$ and $m \geq 4$. We restate Theorem 2 :

Theorem 2. Let $n \geq 3$ and $m \geq 4$. If $G=P_{n} \square C_{m}$, then $G \in \mathcal{M}_{t}$ for some

$$
t \geq \begin{cases}n\left\lfloor\frac{m}{4}\right\rfloor, & \text { if } m \equiv 0,2,3(\bmod 4), \\ n\left\lfloor\frac{m}{4}\right\rfloor-\left\lfloor\frac{n}{2}\right\rfloor, & \text { if } m \equiv 1(\bmod 4) .\end{cases}
$$

Proof. We consider four cases:

Case 1. $m \equiv 0(\bmod 4)$.

Let $I$ be a set $\{(k, i): k \equiv 1(\bmod 2), i \equiv 0(\bmod 4)\}$. The set $I$ is independent and it has size $\frac{m}{4}\left\lfloor\frac{n}{2}\right\rfloor$. Let the graph $H$ arise from $G$ by deleting all vertices in $N_{G}[I]$. The graph $H$ has $\frac{m}{4}$ components, and each one is isomorphic to the graph $H_{n}$. See Figure 2 for an illustration.

By Proposition 11, $H \in \mathcal{M}_{n\left(\frac{m}{4}\right)}$ and

$$
\operatorname{ms}(H)=\left\{\left(\frac{m}{4}\right)\left\lceil\frac{n}{2}\right\rceil, \ldots,\left(\frac{m}{4}\right)\left(\left\lceil\frac{n}{2}\right\rceil+n\right)-2,\left(\frac{m}{4}\right)\left(\left\lceil\frac{n}{2}\right\rceil+n\right)\right\} .
$$

By adding the vertices of $I$, we obtain $\frac{n m}{4}$ different sizes of maximal independent sets in $G$, which are $\left\{\frac{n m}{4}, \ldots, \frac{n m}{2}-2, \frac{n m}{2}\right\}$.

Case 2. $m \equiv 1(\bmod 4)$.

Let $I$ be a set $\{(k, i): k \equiv 1(\bmod 2), i<m-1, i \equiv 0(\bmod 4)\} \cup\{(k, m-4): k \equiv 0(\bmod 4)\} \cup$ $\{(k, m-1): k \equiv 2(\bmod 4)\}$. The set $I$ is independent and it has size $\left(\left(\frac{m-1}{4}\left\lfloor\frac{n}{2}\right\rfloor\right)+\left\lceil\frac{n}{2}\right\rceil\right)$. Let $G^{\prime}$ be the graph arise from $G$ by deleting all vertices in $N_{G}[I]$. The graph $G^{\prime}$ has $\frac{m-1}{4}$ components. 


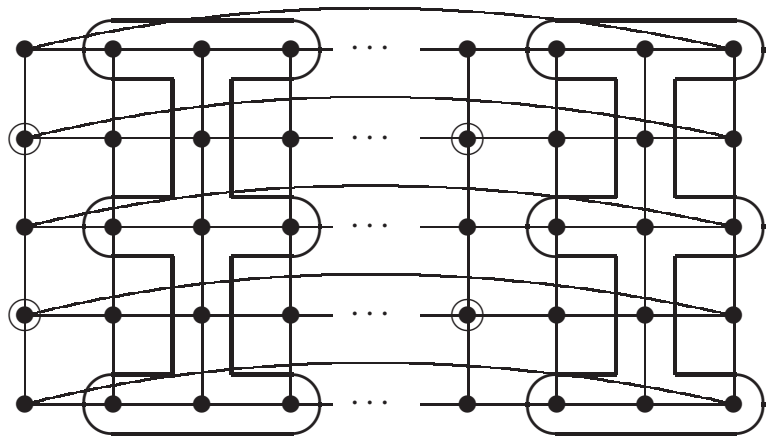

Figure 2: $G=P_{5} \square C_{m}$, with $m \equiv 0(\bmod 4)$. The independent set $I$ is formed by the circled vertices and $G \backslash N_{G}[I]$ has $\frac{m}{4}$ components isomorphic to $H_{5}$.

Denote by $H$ the graph whose components are the $\frac{m-5}{4}$ components of $G^{\prime}$ isomorphic to the graph $H_{n}$. The last component is isomorphic to the graph $F_{n}$. Denote it by $H^{\prime}$. See Figure 3 for an illustration.

By Proposition 11,

$$
\mathrm{m} s(H)=\left\{\left(\frac{m-5}{4}\right)\left\lceil\frac{n}{2}\right\rceil, \ldots,\left(\frac{m-5}{4}\right)\left(\left\lceil\frac{n}{2}\right\rceil+n\right)-2,\left(\frac{m-5}{4}\right)\left(\left\lceil\frac{n}{2}\right\rceil+n\right)\right\} .
$$

By Proposition 12, $\mathrm{m} s\left(H^{\prime}\right)=\left\{\left\lfloor\frac{n}{2}\right\rfloor+1, \ldots, n\right\}$. The union of $I$ with any maximal independent set of $H$ and any independent set of $H^{\prime}$ is a maximal independent set of $G$.

Considering $n \geq 3$, we have that by Proposition 12, $m s\left(H^{\prime}\right)$ has at least two elements. Moreover, $m s(H)$ is an interval with one element missing. Then, $m s(G)=\left\{n\left\lfloor\frac{m}{4}\right\rfloor+\left\lfloor\frac{n}{2}\right\rfloor+1, \ldots, 2 n\left\lfloor\frac{m}{4}\right\rfloor\right\}$ and $|m s(G)|=n\left\lfloor\frac{m}{4}\right\rfloor-\left\lfloor\frac{n}{2}\right\rfloor$.

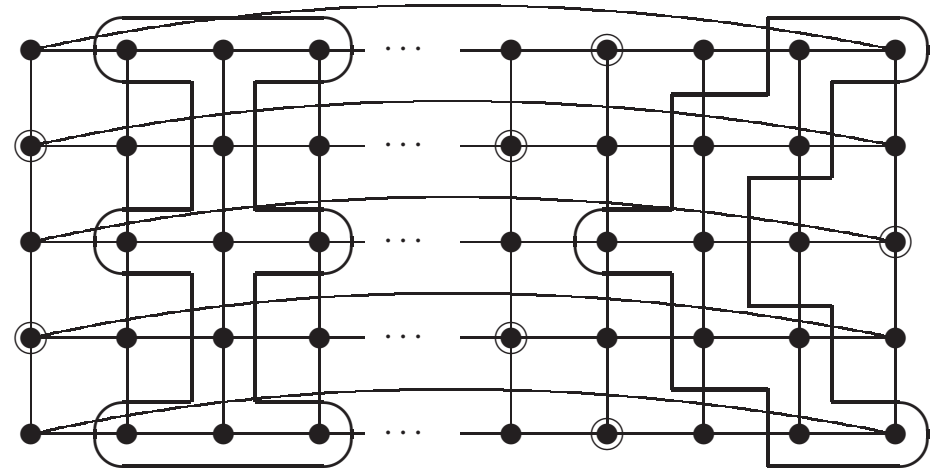

Figure 3: $G=P_{5} \square C_{m}$, with $m \equiv 1(\bmod 4)$. The independent set $I$ is formed by the circled vertices and $G \backslash N_{G}[I]$ has $\frac{m-5}{4}$ components isomorphic to $H_{5}$ and another one isomorphic to $F_{5}$. 
Case 3. $m \equiv 2(\bmod 4)$.

Let $I$ be a set $\{(k, i): k \equiv 1(\bmod 2), i \equiv 0(\bmod 4)\} \cup\{(k, m-1): k \equiv 0(\bmod 2)\}$. The set $I$ is independent and it has size $\left(\left(\frac{m+2}{4}\left\lfloor\frac{n}{2}\right\rfloor\right)+\left\lceil\frac{n}{2}\right\rceil\right)$. Let the graph $H$ arise from $G$ by deleting all vertices in $N_{G}[I]$. The graph $H$ has $\frac{m-2}{4}$ components, each of which is isomorphic to the graph $H_{n}$. See Figure 4(a) for an illustration. By Proposition 11,

$$
\operatorname{ms}(H)=\left\{\left(\frac{m-2}{4}\right)\left\lceil\frac{n}{2}\right\rceil, \ldots,\left(\frac{m-2}{4}\right)\left(\left\lceil\frac{n}{2}\right\rceil+n\right)-2,\left(\frac{m-2}{4}\right)\left(\left\lceil\frac{n}{2}\right\rceil+n\right)\right\} .
$$

By adding the vertices of $I$, we obtain $n\left\lfloor\frac{m}{4}\right\rfloor$ different sizes of maximal independent sets in $G$, which are $\left\{n\left\lceil\frac{m}{4}\right\rceil, \ldots, \frac{n m}{2}-2, \frac{n m}{2}\right\}$.



(a)

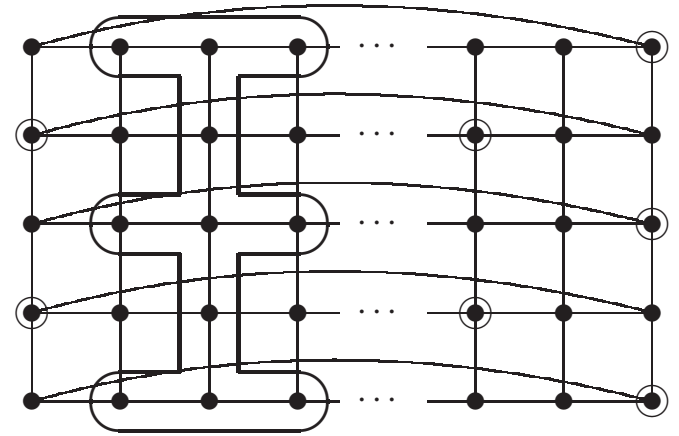

(b)

Figure 4: $G=P_{5} \square C_{m}$, with $m \equiv 2(\bmod 4)(a)$ and $m \equiv 3(\bmod 4)(b)$. The independent set $I$ is formed by the circled vertices and $G \backslash N_{G}[I]$ has $\left\lfloor\frac{m}{4}\right\rfloor$ components isomorphic to $H_{5}$.

Case 4. $m \equiv 3(\bmod 4)$.

Let $I$ be a set $\{(k, i): k \equiv 1(\bmod 2), i \equiv 0(\bmod 4)\} \cup\{(k, m-1): k \equiv 0(\bmod 2)\}$. The set $I$ is independent and it has size $\left(\left(\frac{m+1}{4}\left\lfloor\frac{n}{2}\right\rfloor\right)+\left\lceil\frac{n}{2}\right\rceil\right)$. Let the graph $H$ arise from $G$ by deleting all 
vertices in $N_{G}[I]$. The graph $H$ has $\frac{m-3}{4}$ components, each of which is isomorphic to the graph $H_{n}$. See Figure 4(b) for an illustration. By Proposition 11,

$$
\operatorname{ms}(H)=\left\{\left(\frac{m-3}{4}\right)\left\lceil\frac{n}{2}\right\rceil, \ldots,\left(\frac{m-3}{4}\right)\left(\left\lceil\frac{n}{2}\right\rceil+n\right)-2,\left(\frac{m-3}{4}\right)\left(\left\lceil\frac{n}{2}\right\rceil+n\right)\right\} .
$$

By adding the vertices of $I$, we obtain $n\left\lfloor\frac{m}{4}\right\rfloor$ different sizes of maximal independent sets in $G$, which are $\left\{n\left\lceil\frac{m}{4}\right\rceil, \ldots, n\left\lfloor\frac{m}{2}\right\rfloor-2, n\left\lfloor\frac{m}{2}\right\rfloor\right\}$.

\section{CONCLUDING REMARKS}

Since a vertex in a cylindrical grid graph $G$ can dominate at most 5 vertices, a lower bound for $i(G)$ is $\left\lceil\frac{n m}{5}\right\rceil$, which gives us an upper bound $n\left\lfloor\frac{m}{2}\right\rfloor-\left\lceil\frac{n m}{5}\right\rceil+1$ for the quantity of different sizes of maximal independent sets in $G$. Furthermore, the bound in Theorem 2 is sharp when $m$ is a multiple of 4 and $n$ is a small value. The technique we have applied may be useful to find maximal independent sets of different sizes in other graph classes or even in the study of other problems in Graph Theory.

\section{ACKNOWLEDGEMENTS}

Márcia R. Cappelle was partially suported by the Fundação de Amparo à Pesquisa do Estado de Goiás (FAPEG). We would like to thank the anonymous reviewers for the very useful comments and suggestions.

RESUMO. Se um grafo $G$ tem exatamente $t$ tamanhos diferentes de conjuntos independentes maximais, $G$ pertence a uma coleção chamada $\mathcal{M}_{t}$. Para o produto Cartesiano do grafo $P_{n}$, o caminho de tamanho $n$, e $C_{m}$, o ciclo de tamanho $m$, chamado grade cilíndrica, apresentamos um método para encontrar conjuntos independentes maximais com diferentes tamanhos e um limite inferior para $t$, tal que estes grafos pertençam à $\mathcal{M}_{t}$.

Palavras-chave: Grafo bem-coberto, conjunto independente maximal, produto Cartesiano.

\section{REFERENCES}

[1] R. Barbosa \& B.L. Hartnell. Some problems based on the relative sizes of the maximal independent sets in a graph. Congr. Numerantium, 131 (1998), 115-121.

[2] R. Barbosa \& B.L. Hartnell. The effect of vertex and edge deletion on the number of sizes of maximal independent sets. J. Combin. Math. Combin. Comput., 70 (2009), 111-116.

[3] R. Barbosa, M.R. Cappelle \& D. Rautenbach. On Graphs with Maximal Independents Sets of Few Sizes, Minimum Degree at least 2, and Girth at least 7. Discrete Math., 313 (2013), 1630-1635.

[4] A. Finbow, B. Hartnell \& C. Whitehead. A characterization of graphs of girth eight or more with exactly two sizes of maximal independent sets. Discrete Math., 125 (1994), 153-167. 
[5] A.O. Fradkin. On the well-coveredness of Cartesian products of graphs. Discrete Math., 309(1) (2009), 238-246.

[6] B.L. Hartnell \& D.F. Rall. On graphs having maximal independent sets of exactly $t$ distinct cardinalities. Graphs and Combinatorics, 29(3) (2013), 519-525.

[7] B.L. Hartnell \& D.F. Rall. On the Cartesian product of non well-covered graphs. Eletr. J. Comb., 20(2) (2013), P21.

[8] M. Nandi, S. Parui \& A. Adhikari. The domination numbers of cylindrical grid graphs Applied Math. and Comp., 217(10) (2011), 4879-4889.

[9] M.D. Plummer. Well-covered graphs, J. Combin. Theory, 8 (1970), 91-98.

[10] M.D. Plummer. Well-covered graphs: a survey, Quaestiones Math., 16 (1993), 253-287.

[11] J. Topp \& L. Volkmann. On the well coveredness of Products of Graphs. Ars Combinatoria, 33 (1992), 199-215. 